\title{
Evaluation and comparison of outcomes in dogs with periarticular and nonperiarticular histiocytic sarcoma
}

\author{
Shawna L. Klahn, DVM; Barbara E. Kitchell, DVM, PhD, DACviM; Nikolaos G. Dervisis, DVM, PhD, DACVIM
}

\begin{abstract}
Objective-To evaluate and compare the outcomes of dogs with periarticular histiocytic sarcoma (PAHS) and histiocytic sarcoma of other anatomic locations (non-PAHS) and identify factors associated with outcome for dogs with PAHS.

Design-Retrospective cohort study.

Animals - 19 dogs with PAHS and 31 dogs with non-PAHS.

Procedures-Medical records of dogs with histiocytic sarcoma that underwent definitive local treatment (surgery or radiation), chemotherapy, or a combination of these were reviewed. Patient signalment, clinical signs, staging test results, clinicopathologic data, type of treatment, response, and outcome were collected, and potential risk factors in dogs with PAHS were identified and analyzed for an association with outcome.
\end{abstract}

Results-Dogs with PAHS lived significantly longer than did dogs with non-PAHS, with an overall median survival times of 391 (range, 48 to 980) and 128 (range, 14 to 918) days, respectively, despite the presence of suspected metastasis at diagnosis in 13 of 19 dogs with PAHS. Dogs with PAHS without evidence of metastasis at diagnosis lived significantly longer than did dogs with PAHS with evidence of metastasis, with median survival times of 980 (range, 83 to 980) and 253 (range, 48 to 441) days, respectively. Administration of prednisone in dogs with PAHS was associated with a significantly shorter time to tumor progression (TTP) and increased risk of tumor progression and death.

Conclusions and Clinical Relevance-Results indicated that dogs with PAHS may have a favorable outcome independent of metastatic status when treated with chemotherapy or aggressive multimodal treatment. The concurrent administration of prednisone may be a negative predictive factor for survival time and TTP in dogs with PAHS. (J Am Vet Med Assoc 2011;239:90-96)

$\mathrm{H}$ istiocytic sarcoma is a round-cell neoplasm associated with a poor prognosis in dogs. Advances in veterinary pathology and immunohistochemical techniques have enabled identification of the putative cell of origin and have resulted in the reclassification of HS in dogs. ${ }^{1,2}$ Currently, there are 3 recognized clinical manifestations of HS. Hemophagocytic HS is thought to arise from macrophages of the splenic red pulp and bone marrow and is definitively diagnosed by positive CD18 and CD11d antibody immunohistochemical staining. Clinically, it is characterized by diffuse splenomegaly, regenerative anemia, thrombocytopenia, hypoalbuminemia, and hypocholesterolemia. Hemophagocytic HS follows a rapidly progressive clinical course despite treatment; the reported MST is approximately 7 weeks. ${ }^{1}$ Localized HS and disseminated HS are thought to arise from myeloid dendritic antigen-presenting cells. The 2 are morphologically and immunohistochemically identical, with positive staining results for CD18 and CD11c antibodies and negative results for CD11d antibodies, but are distinguished on the basis of clinical presentation. ${ }^{2}$ Localized HS appears to arise from a single site, typically

From the Department of Small Animal Clinical Sciences, College of Veterinary Medicine, Michigan State University, East Lansing, MI 48824.

Presented in part as an oral presentation at the 29th Annual Conference of the Veterinary Cancer Society, Austin, Tex, October 2009.

Address correspondence to Dr. Klahn (klahn@cvm.msu.edu).

\begin{tabular}{|c|c|}
\hline \multicolumn{2}{|r|}{ Abbreviations } \\
\hline $\mathrm{AC}$ & $\begin{array}{l}\text { Doxorubicin, cyclophosphamide } \\
\text { chemotherapy protocol }\end{array}$ \\
\hline $\mathrm{CHOP}$ & $\begin{array}{l}\text { Cyclophosphamide, doxorubicin, } \\
\text { vincristine, prednisone chemotherapy } \\
\text { protocol }\end{array}$ \\
\hline HS & Histiocytic sarcoma \\
\hline LHS & Localized histiocytic sarcoma \\
\hline MST & Median survival time \\
\hline PAHS & Periarticular histiocytic sarcoma \\
\hline TTP & Time to tumor progression \\
\hline VAC & $\begin{array}{l}\text { Vincristine, doxorubicin, cyclophosphamide } \\
\text { chemotherapy protocol }\end{array}$ \\
\hline
\end{tabular}

involving the lung, skin, subcutaneous tissues, bones, or joints. ${ }^{2-6}$ Disseminated HS involves multiple organ systems, which could indicate metastasis and progression of the localized form. Alternatively, disseminated HS may be a primary multicentric disease, in which tumors arise simultaneously in multiple locations. ${ }^{2}$

Despite reported metastatic rates of $70 \%$ to $91 \%$, treatment consisting of surgery, radiation, chemotherapy, or a combination of these appears to improve outcome in dogs with HS. ${ }^{3,4}$ In a recent study ${ }^{7}$ of 59 dogs with gross or disseminated disease, $54 \%$ of dogs that lived longer than 7 days following the first treatment with lomustine achieved either a partial or complete response, and 
the patients that responded experienced an MST of 5.5 months. In a series ${ }^{4}$ of 37 Flat-Coated Retrievers with LHS, the presence of distant metastasis at diagnosis was a strong negative predictor of survival time. However, any form of treatment beyond pain management or corticosteroids significantly improved outcome regardless of metastatic status. Thirty-four of the patients had disease involving an appendicular joint, ${ }^{4}$ and prior reports ${ }^{3-5}$ indicate that a periarticular subset of LHS may exist. Eighteen of 35 dogs in which synovial cell sarcomas were previously diagnosed were reclassified as having $\mathrm{HS}$ on the basis of CD18 immunohistochemical expression. ${ }^{3}$ Three of the dogs in that study ${ }^{3}$ received aggressive multimodal treatment consisting of amputation and adjuvant chemotherapy and had a mean survival duration of 9 months. More recently, long-term survival in a series of 16 dogs with LHS and no measurable residual disease was reported. ${ }^{6}$ In that study, aggressive multimodal treatment consisting of definitive local treatment and lomustine chemotherapy administered in an adjuvant setting resulted in an MST of 568 days. ${ }^{6}$

On the basis of prior reports, we hypothesized that dogs with PAHS would have a longer survival time than would dogs with HS of other anatomic locations (nonPAHS), independent of metastatic disease presence, when both groups received treatment that consisted of chemotherapy, surgery, radiation, or a combination of these. The primary objective of the present study was to evaluate and compare the outcomes of dogs with PAHS and non-PAHS. The secondary objective was to identify factors associated with survival time or TTP for dogs with PAHS.

\section{Materials and Methods}

Animals-The medical records of the Veterinary Teaching Hospital at Michigan State University were searched for dogs in which HS had been diagnosed between 2004 and 2008. For inclusion in the study, dogs had a cytologic or histopathologic diagnosis of HS; received treatment consisting of surgery, radiation therapy, chemotherapy, or a combination; lived longer than 7 days following first treatment; and had adequate follow-up to assess response. The time interval between patient visits and follow-up was determined by the treatment protocol.

Chemotherapy protocols consisted of lomustine or a doxorubicin-based protocol. Lomustine (60 to $80 \mathrm{mg} /$ $\mathrm{m}^{2} \mathrm{PO}, \mathrm{q} 3 \mathrm{wk}$ ) was administered as a 3-week cycle indefinitely until progressive disease or unacceptable toxicity was noted. All doxorubicin-based protocols were scheduled as 3-week cycles. Patients were administered consecutive cycles according to the protocol until progressive disease or unacceptable toxicity occurred, for a maximum of 6 cycles ( 18 weeks). Doxorubicin as a single agent was administered at $30 \mathrm{mg} / \mathrm{m}^{2}$, IV, every 3 weeks. Other doxorubicin-based protocols included AC (doxorubicin, $30 \mathrm{mg} / \mathrm{m}^{2}$, IV, on day l; cyclophosphamide, $50 \mathrm{mg} /$ $\mathrm{m}^{2}, \mathrm{PO}, \mathrm{q} 24 \mathrm{~h}$ on days $1,2,3$, and 4), VAC (doxorubicin, $30 \mathrm{mg} / \mathrm{m}^{2}$, IV, on day 1 ; cyclophosphamide, $50 \mathrm{mg} / \mathrm{m}^{2}$, PO, q $24 \mathrm{~h}$ on days $1,2,3$, and 4; vincristine, $0.5 \mathrm{mg} / \mathrm{m}^{2}$, IV, on days 8 and 15), and CHOP (doxorubicin, $30 \mathrm{mg} / \mathrm{m}^{2}$, $\mathrm{IV}$, on day 1 ; vincristine, $0.5 \mathrm{mg} / \mathrm{m}^{2}$, IV, on days 8 and 15 ; cyclophosphamide, $50 \mathrm{mg} / \mathrm{m}^{2}$, PO, q $48 \mathrm{~h}$; prednisone, 20 $\mathrm{mg} / \mathrm{m}^{2}$, PO, q $24 \mathrm{~h}$ for 7 days, followed by every other day administration).
Selected patients were assigned to 2 groups on the basis of involvement of an appendicular joint. In an effort to exclude dogs with the hemophagocytic form of HS, patients were excluded from the study if they did not survive beyond 7 days following the date of first treatment. Patients were also excluded if they received palliative care consisting solely of medical pain management, coarsefractionated radiation therapy (three 8-Gray or six 6-Gray fractions), corticosteroids, or a combination of these. Data were collected by review of medical records and follow-up telephone calls to referring veterinarians when necessary. Information obtained included signalment, clinical signs, method and date of diagnosis, results of initial clinicopathologic and staging tests (3-view thoracic radiography and abdominal ultrasonography), type of treatment, outcome of treatment, response duration, and cause and date of death. The presence or absence of metastatic disease was determined by use of radiography or ultrasonography and confirmed with fine-needle aspiration cytology when possible.

Evaluation of response-Tumor response was determined each time dogs were examined. Complete response was defined as 100\% reduction in measurable tumor volume for at least 21 days, partial response was defined as $>50 \%$ but $<100 \%$ reduction in measurable tumor volume for at least 21 days, stable disease was defined as $\leq 50 \%$ reduction but $<20 \%$ increase in tumor volume for at least 21 days, and progressive disease was defined as $\geq 20 \%$ increase in tumor volume or the appearance of new lesions. Transient decreases in measurable disease that persisted for $<21$ days followed by increased tumor size were defined as progressive disease.

Statistical analysis-The time to diagnosis was calculated from the date of first known onset of clinical signs to the date of first examination at the Veterinary Teaching Hospital. An independent samples $t$ test was used to test for differences in times to diagnosis between the PAHS and non-PAHS groups.

Survival time was calculated from the date of first treatment to the date of death, and TTP was calculated from the date of first treatment to the date of documented local tumor recurrence or distant metastasis. Survival times for dogs lost to follow-up were censored on the date they were last known to be alive. Survival times for dogs that died of another cause were censored on the date of death. Time to tumor progression for dogs lost to follow-up was censored on the date they were last reported to be alive. The KaplanMeier survival analysis method was used to estimate TTP and survival time curves following treatment. The log rank test was used to compare the impact of potential risk factors (Hct, platelet count, albumin concentration, concurrent prednisone administration, type of treatment used, and presence of locoregional or distant metastasis) on TTP and survival time. The Cox proportional hazards regression method was used to determine whether the potential risk factors were associated with TTP or survival time. The potential risk factors were entered in the regression model in a forward fashion if the value of $P$ was $<0.05$ and were removed if the $P$ value was $>0.1$.

Two-sided $P$ values are reported, with values of $P<$ 0.05 considered significant. Statistical analyses were performed with standard software. ${ }^{a}$ 


\section{Results}

Patient characteristics-Fifty dogs were treated for HS between 2004 and 2008. Nineteen (38\%) dogs had disease involving an appendicular joint and were categorized as having PAHS, and $31(62 \%)$ dogs had disease not involving an appendicular joint and were categorized as having non-PAHS.

The mean \pm SD age at diagnosis of the 19 dogs with PAHS was $8.8 \pm 1.8$ years. Three dogs were sexually intact males, 7 were castrated males, 1 was a sexually intact female, and 8 were spayed females. There were 4 Labrador Retrievers, 4 Golden Retrievers, 3 Rottweilers, 2 Flat-Coated Retrievers, and 6 other purebred dogs. The mean weight was $35.2 \pm 9.4 \mathrm{~kg}(77.4 \pm 20.7 \mathrm{lb})$.

The mean age at diagnosis of the 31 dogs with nonPAHS was $7.8 \pm 3.0$ years, and the mean weight was $32.0 \pm 14 \mathrm{~kg}(70.4 \pm 30.8 \mathrm{lb})$. There were 7 Golden Retrievers, 6 Labrador Retrievers, 4 Bernese Mountain Dogs, 3 mixed-breed dogs, and 11 other purebred dogs. There was no significant difference in the age or weight between the non-PAHS and PAHS groups.

Dogs with PAHS had lameness (15 [79\%]) or a soft tissue swelling (13 [68\%]) on initial examination. Peripheral lymphadenopathy was the predominant finding on initial examination in $1 \mathrm{dog}$. The mean time to diagnosis was $126 \pm 81$ days (median, 98 days; range, 15 to 282 days). Primary tumor lesions were located in the stifle (7 [37\%]), elbow (5 [26\%]), and shoulder (4 [21\%]) joints, and 1 each involved the hip, carpal, and tarsal joints (Table 1). Seventeen of the dogs were evaluated with regional radiographs, and $10(58.8 \%)$ dogs had radiographic evidence of osteolysis or proliferation. Of these 10 patients, the lesion was described as osteolytic in 5 dogs, osteolytic and proliferative in 2 dogs, osteolytic and proliferative but primarily osteolytic in 2 dogs, and irregular in $1 \mathrm{dog}$. The majority of tumors were diagnosed by use of histopathology (16 [84\%] dogs) and confirmed with immunohistochemical staining for CD18 antibodies in most of these patients (14 [87.5\%] dogs). Seventeen dogs were evaluated with thoracic radiography and abdominal ultrasonography, and 2 dogs were evaluated with either thoracic radiography or abdominal ultrasonography. Fine-needle aspiration cytology of a regional lymph node was performed in 9 (47\%) dogs. Most dogs were suspected to have evidence of metastatic disease at diagnosis $(n=$ 13 [68\%]). Seven dogs had locoregional metastases to lymph nodes only, and 6 (31.3\%) dogs also had evi- dence of distant or visceral metastases involving the lung $(\mathrm{n}=2)$, the liver (2), and 1 each in the kidney or spleen. Complete blood counts and results of serum biochemical analysis were available for all dogs. Mild hypoalbuminemia was noted in $8(42 \%)$ dogs (mean, $2.3 \pm 0.3 \mathrm{~g} / \mathrm{dL}$; median, $2.4 \mathrm{~g} / \mathrm{dL}$ [range, 1.9 to $2.7 \mathrm{~g} /$ $\mathrm{dL}$; reference range, 2.8 to $4.0 \mathrm{~g} / \mathrm{dL}]$ ). Mild anemia was noted in $6(32 \%)$ dogs (mean, $37.6 \pm 2.2 \%$; median, $37.5 \%$ [range, $35 \%$ to $40.7 \%$ ]; reference range, $41 \%$ to $55 \%])$. One dog also had thrombocytopenia (51,000 X $10^{3} / \mu \mathrm{L}$; reference range, 155 to $393 \times 10^{3} / \mu \mathrm{L}$ ).

Dogs in the non-PAHS group had nonspecific or site-specific clinical signs on initial examination, including inappetance $(\mathrm{n}=14[45 \%])$, vomiting and diarrhea (8 [26\%]), lethargy (8 [26\%]), coughing and dyspnea (10 [32\%]), skin masses (6 [19\%]), or an abdominal mass (4 [13\%]). The time to diagnosis was significantly shorter than that of PAHS dogs (mean, $65 \pm 95$ days; median, 30 days [range, 3 to 446 days]; $P=0.0246])$. The most common tumor locations were lymph nodes ( $\mathrm{n}=13[42 \%])$, lung (10 [32\%]), skin (6 [19\%]), spleen (6 [19\%]), and liver (5 [16\%]). Most dogs $(n=22[71 \%])$ had tumors in more than 1 location (ie, evidence of metastasis). Diagnosis was obtained by use of histopathology in the majority of the patients ( $\mathrm{n}$ $=20[64.5 \%])$ and confirmed with immunohistochemical staining for CD18 antibodies in most of these patients (14 [70\%]). Mild hypoalbuminemia (mean, 2.4 $\pm 0.2 \mathrm{~g} / \mathrm{dL})$ was noted in $17(55 \%)$ dogs, mild anemia (mean, $33.6 \pm 7.9 \%$ ) was noted in $8(26 \%)$ dogs, and thrombocytopenia was noted in $5(16 \%)$ dogs (mean, $\left.101,800 \pm 49,200 \times 10^{3} / \mu \mathrm{L}\right)$ at the time of diagnosis.

Treatment-Definitive local treatment was performed in 11 of 19 dogs with PAHS: 10 dogs underwent amputation, and 1 dog was treated with excisional surgery followed by curative-intent radiation therapy. Radiation therapy was administered with a linear accelerator $^{\mathrm{b}}$ with $10 \mathrm{MV}$ of energy. The prescription consisted of 18 daily (Monday through Friday) fractions of $3 \mathrm{~Gy}$, with the treatment field including the primary tumor and the regional lymph node. Eight of these 11 dogs received adjuvant chemotherapy. Seven dogs received lomustine, and 1 dog was treated with VAC. Eight of 19 dogs received chemotherapy as sole treatment, with 4 dogs receiving lomustine and 4 dogs receiving a doxorubicin-based protocol (2 VAC, 1 AC, and 1 CHOP).

Five of 19 dogs with PAHS received prednisone as part of the treatment protocol. Of these 5 dogs, 1

Table 1-The breed, primary site, treatment, and metastatic status of 19 dogs with PAHS.

\begin{tabular}{|c|c|c|c|c|c|c|c|c|}
\hline \multirow[b]{2}{*}{ Breed } & \multicolumn{4}{|c|}{ Primary site (joint) } & \multicolumn{3}{|c|}{ Treatment } & \multirow[b]{2}{*}{$\begin{array}{l}\text { Metastasis } \\
\text { suspected }\end{array}$} \\
\hline & Stifle & Elbow & Shoulder & $\begin{array}{c}\text { Other } \\
\text { appendicular } \\
\text { joint* }\end{array}$ & $\begin{array}{l}\text { Local } \\
\text { therapy } \\
\text { alone }\end{array}$ & $\begin{array}{c}\text { Chemotherapy } \\
\text { alone }\end{array}$ & $\begin{array}{l}\text { Local therapy } \\
\text { + adjuvant } \\
\text { chemotherapy }\end{array}$ & \\
\hline Labrador Retriever $(n=4)$ & 1 & 0 & 2 & 1 & 0 & 2 & 2 & 2 \\
\hline Golden Retriever $(n=4)$ & 2 & 1 & 0 & 1 & 1 & 1 & 2 & 3 \\
\hline Rottweiler $(n=3)$ & 1 & 1 & 0 & 1 & 1 & 1 & 1 & 1 \\
\hline Flat-Coated Retriever ( $n=2$ ) & 1 & 1 & 0 & 0 & 0 & 2 & 0 & 2 \\
\hline Other purebred dogst $(n=6)$ & 2 & 1 & 3 & 0 & 0 & 2 & 4 & 5 \\
\hline Total $(n=19)$ & 7 & 4 & 5 & 3 & 2 & 8 & 9 & 13 \\
\hline
\end{tabular}


received prednisone as treatment for concurrent lymphoma $\left(0.77 \mathrm{mg} / \mathrm{m}^{2}\right.$, PO, q $\left.24 \mathrm{~h}\right), 1$ as treatment for suspected immune-mediated thrombocytopenia (1.9 $\mathrm{mg} / \mathrm{m}^{2}$, PO, q $24 \mathrm{~h}$ ), and 1 as part of the CHOP protocol $\left(20 \mathrm{mg} / \mathrm{m}^{2}\right.$, PO, q $24 \mathrm{~h}$, for 7 days, followed by every other day). Two dogs received prednisone concurrently with lomustine (prednisone dosages $1.3 \mathrm{mg} /$ $\mathrm{kg}[0.59 \mathrm{mg} / \mathrm{lb}], \mathrm{PO}, \mathrm{q} 24 \mathrm{~h}$, and $1.5 \mathrm{mg} / \mathrm{kg}$ [0.68 mg/ lb], PO, q 24 h).

Of dogs in the non-PAHS group, 8 of 31 received definitive local treatment, consisting of splenectomy (n $=4$ ), lung lobectomy (2), liver lobectomy (1), or curative-intent radiation therapy (1). Radiation therapy was administered with a linear accelerator ${ }^{\mathrm{b}}$ with $6 \mathrm{MV}$ of energy. The prescription consisted of 18 daily (Monday through Friday) fractions of $3 \mathrm{~Gy}$, and the treatment field did not include the regional lymph node. Six of these 8 dogs were treated with chemotherapy in an adjuvant setting (4 lomustine, 1 VAC, and 1 CHOP). The remaining 23 dogs were treated solely with chemotherapy (18 lomustine, 3 doxorubicin as a single agent, 1 $\mathrm{AC}$, and $1 \mathrm{CHOP}$ ).

Patient follow-up, assessment, and causes of death for all dogs-Patients were examined, and the tumor response was evaluated at each visit. The time interval between patient visits for all dogs varied on the basis of the type of treatment and chemotherapy protocol selected, ranging from 1 to 3 weeks. The majority of dogs (44/50 [88\%]) received treatment at the Veterinary Teaching Hospital of Michigan State University up to or within 3 months of death. Of these 44 (88\%) dogs, 38 (86\%) died from the disease and $6(14 \%)$ died of unknown causes. Of the $6(14 \%)$ dogs that survived longer than 3 months following the last visit to Michigan State University, 5 (83\%) dogs had PAHS and 1 (17\%) dog had non-PAHS. Of the 5 dogs with PAHS, 3 dogs died of unknown causes at 5 , 8 , and 19 months after the last visit to the hospital, 1 dog was reported to have died because of disease 919 days after the last treatment with chemotherapy, and 1 dog died 267 days after undergoing amputation with no evidence of HS on necropsy. The dog with non-PAHS had pulmonary LHS, did not receive adjuvant treatment, and was euthanatized because of diabetes mellitus and suspected hyperadrenocorticism 918 days following lung lobectomy.

Patient outcomes-Nine of the 19 dogs with PAHS had gross measurable disease, and response to treatment could be evaluated. Two dogs achieved a complete response, 3 achieved a partial response, 2 had stable disease, and 2 had progressive disease, resulting in an overall response rate of $56 \%(5 / 9)$. The median duration of response for the dogs that responded was 244 days (range, 63 to 267 days), and the MST for responders was 428 days (range, 134 to 441 days) versus 116 days (range, 46 to 133 days) for dogs with stable disease or progressive disease. The median TTP for the 10 dogs without measurable disease was 162 days (range, 56 to 490 days), and these dogs survived a median of 510 days (range, 83 to 980 days). At the time of writing, 18 dogs had died and 1 dog was lost to followup. Thirteen of the 18 dogs died or were euthanatized because of progressive disease, 4 were euthanatized for unknown reasons, and 1 dog died of another cause at
267 days with no evidence of local recurrence or metastatic disease on necropsy.

Twenty-three of 31 dogs with non-PAHS had gross measurable disease, and their response to chemotherapy could be evaluated. The overall response rate was 35\% (8/23), with 2 dogs achieving a complete response, 6 achieving a partial response, 5 having stable disease, and 10 having progressive disease. The median duration of response for the 8 dogs that responded was 119 days (range, 14 to 161 days), and the MST for responders was 140 days (range, 97 to 470 days) versus 48 days (range, 14 to 424 days) for dogs with stable disease or progressive disease. The median TTP for the remaining 8 dogs that did not have measurable disease was 260 days (range, 27 to 918 days), and they survived a median of 400 days (range, 27 to 918 days). At the time of writing, 27 dogs had died and 4 dogs were lost to follow-up. Twenty-six of the 27 dogs died or were euthanized because of progressive disease.

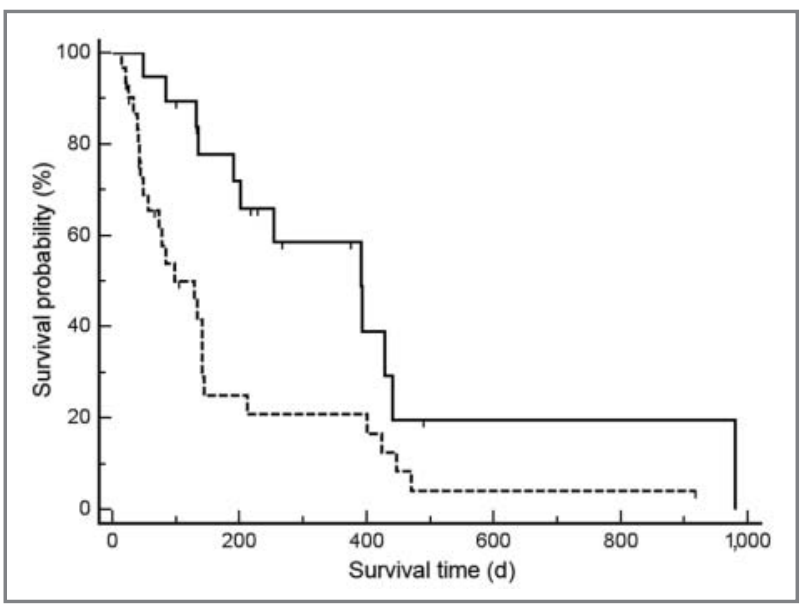

Figure 1-Kaplan-Meier survival curves estimating MST for dogs with PAHS (solid line; $n=19$ ) versus non-PAHS (dashed line; 31). Dogs with PAHS had a significantly $(P=0.008)$ longer MST of 391 days, compared with 128 days for dogs with non-PAHS. Vertical tick marks represent animals that were censored.

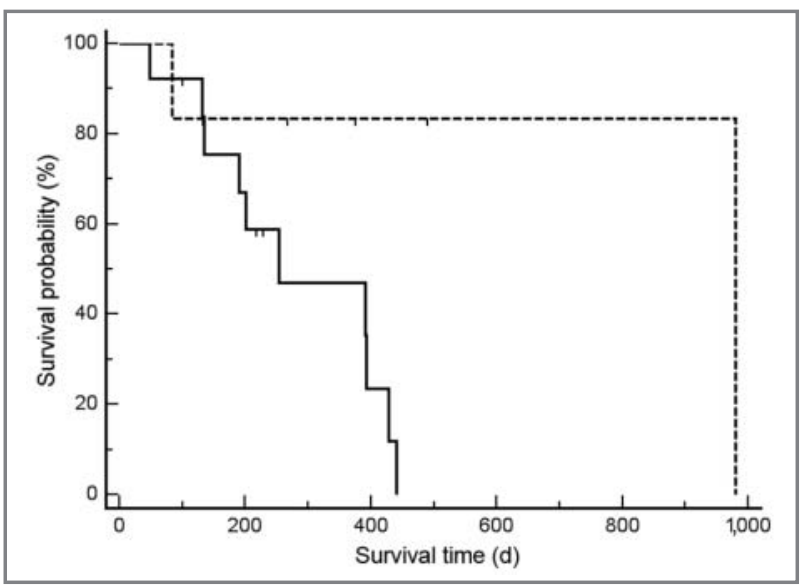

Figure 2-Kaplan-Meier survival curves estimating MST in dogs with PAHS and evidence of metastatic disease at diagnosis (solid line; $n=13$ ) versus dogs with PAHS without evidence of metastatic disease at diagnosis (dashed line; 6). Dogs with PAHS that had evidence of metastatic disease at diagnosis had a significantly $(P=0.045)$ shorter MST ( 253 days) than dogs without evidence of metastasis (980 days). Vertical tick marks represent animals that were censored. 


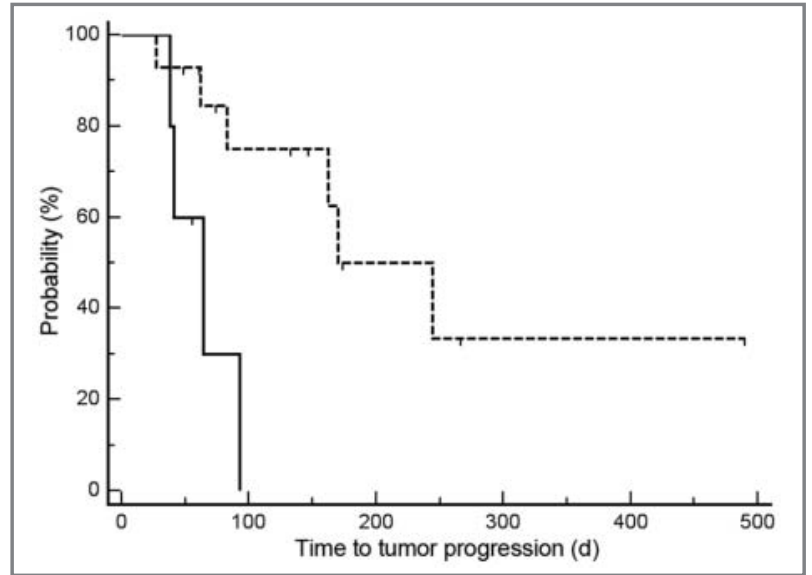

Figure 3-Kaplan-Meier survival curves estimating the median TTP in dogs with PAHS receiving concurrent prednisone treatment (solid line; $n=5$ ) versus dogs with PAHS that did not receive concurrent prednisone treatment (dashed line; 14). Dogs with PAHS that received prednisone had a significantly $(P=$ 0.008 ) shorter TTP (median, 64 days) than did dogs that did not receive prednisone (median, 244 days). Vertical tick marks represent animals that were censored.

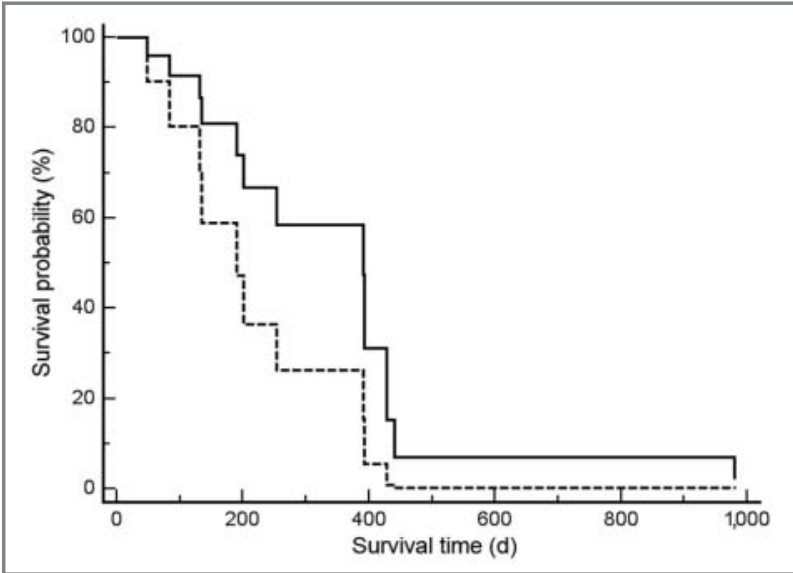

Figure 4-Cox proportional hazard function curves of survival time for dogs with PAHS that received concurrent prednisone treatment (dashed line; $n=5$ ) and dogs with PAHS that did not receive prednisone treatment (solid line, 14; hazard ratio, 4.75; $P=0.033$ )

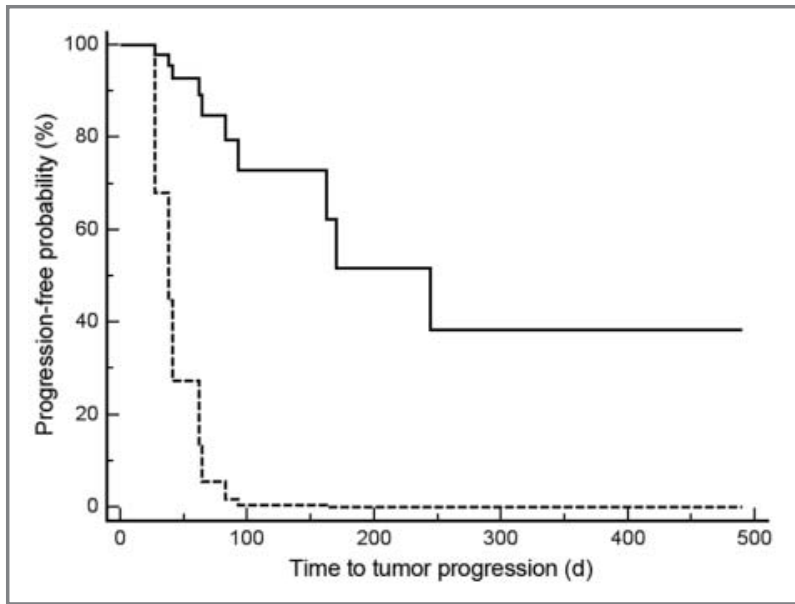

Figure 5-Cox proportional hazard function curves of TTP for dogs with PAHS that received concurrent prednisone treatment (dashed line; $n=5$ ) and dogs with PAHS that did not receive concurrent prednisone treatment (solid line, 14; hazard ratio, 6.29; $P=0.019$ ).
One dog with pulmonary HS and no evidence of metastatic disease at diagnosis was treated with lung lobectomy and euthanized because of diabetes mellitus and suspected hyperadrenocorticism at 918 days.

Comparison of outcome for PAHS and non-PAHS groups-Dogs with PAHS survived significantly longer than did dogs with non-PAHS, with an MST of 391 days (range, 48 to 980 days), compared with 128 days (range, 14 to 470 days; $P=0.008$; Figure 1 ). The median TTP was also longer in dogs with PAHS versus dogs with non-PAHS, at 162 days (range, 27 to 244 days) versus 71 days (range, 14 to 343 days), although this difference was not significant $(P=0.056)$.

Risk factor analysis for dogs with PAHS-Univariate analysis identified 2 factors significantly predictive of patient outcome in dogs with PAHS. Dogs without evidence of metastasis at the time of diagnosis had an MST of 980 days (range, 83 to 980), compared with 253 days (range, 48 to 441) in dogs with evidence of metastasis $(P=0.045$; Figure 2$)$. Dogs receiving prednisone as concurrent treatment had a median TTP of 64 days (range, 38 to 93 ) versus 244 days (range, 27 to 490 ) in dogs that did not receive prednisone $(P=0.008$; Figure 3$)$. Hematocrit, albumin concentration, platelet count, and treatment type were not predictive of survival time or TTP.

The Cox proportional hazards model indicated that concurrent administration of prednisone was a negative predictive factor for survival time and TTP. Concurrent administration of prednisone was associated with an increased risk of death (hazard ratio, $4.75 ; P=0.033$; Figure 4 ) and an increased risk of tumor progression (hazard ratio, 6.29; $P=0.019$; Figure 5).

\section{Discussion}

The results of the present study indicated that dogs with PAHS may have a favorable outcome independent of metastatic status when treated with chemotherapy or aggressive multimodal treatment. The concurrent administration of prednisone may be a negative predictive factor for survival time and TTP in dogs with PAHS. In dogs, HS is typically associated with high metastatic potential and grave prognosis, ${ }^{2-4,7}$ and previous reports ${ }^{3,4,6}$ have encouraged the use of aggressive multimodal treatment to improve outcome. Recently, the MST for dogs with LHS in which tumors were reduced to microscopic disease with definitive local treatment followed by adjuvant lomustine was reported ${ }^{6}$ to be approximately 19 months. In the present study, dogs in both the PAHS and non-PAHS groups that did not have evidence of measurable disease following definitive local treatment had MSTs that exceeded 1 year (510 [range, 83 to 980 ] and 400 [range, 27 to 918] days, respectively). Historically, the majority of dogs with HS have disseminated disease at the time of initial examination, with reported metastatic rates of $70 \%$ to $91 \% .^{3-5,7}$ Our findings are similar, and in both the PAHS and nonPAHS groups, nearly $70 \%$ of dogs were suspected to have metastatic disease. Despite the presence of metastatic disease, outcome may be improved when patients are treated with surgery, radiation, chemotherapy, or a combination of these. Lomustine has been reported ${ }^{7}$ 
to have antineoplastic activity against HS. In the presence of gross evidence of disease, $46 \%$ of dogs with HS achieved a response to treatment, and the reported MST for responders was 172 days. $^{7}$ In a series ${ }^{4}$ of 37 FlatCoated Retrievers with HS, the overall response rate of dogs receiving lomustine was $66.7 \%$. The majority of dogs in that series had HS arising near an appendicular joint. In the present study, the non-PAHS group had a $35 \%(8 / 23)$ overall response rate, with responders living a median of 140 days (range, 97 to 470 days). In the present study, nearly half of the dogs in the PAHS group received chemotherapy as sole treatment, achieving an overall response rate of 56\% (5/9). The MST for dogs with PAHS that responded to chemotherapy was similar to that of dogs with PAHS that did not have evidence of measurable disease after definitive local treatment (428 [range, 134 to 441] and 510 [range, 83 to 980] days, respectively). These results suggest that dogs with PAHS may have a favorable outcome independent of metastatic status when treated with chemotherapy or aggressive multimodal therapy.

Localized HS arising near an appendicular joint has been reported in Rottweilers, 2,3,5,6 Labrador Retrievers, ${ }^{2,3,5,6}$ Golden Retrievers, ${ }^{2,3,5,6}$ and Flat-Coated Retrievers $^{2-6}$ and may represent a subtype of LHS. In a report by Craig et al, ${ }^{3} 18$ tumors previously diagnosed as synovial cell sarcomas were reclassified as HS, and the overall MST for all 18 dogs was 5.3 months, but the treatments administered varied within this group. ${ }^{3}$ Nine of the dogs in that study ${ }^{3}$ underwent amputation, and 3 received adjuvant chemotherapy, for which the mean survival time was 9 months. Fidel et $\mathrm{al}^{4}$ described HS in Flat-Coated Retrievers, with 34 of the 37 tumors located in the periarticular region. The dogs in that series received a variety of treatments, including palliative care. For dogs that received treatment consisting of surgery, radiation, chemotherapy, or a combination of these, the MST was significantly prolonged at 167 days, compared with 30 days for palliation. ${ }^{4}$ Although a comparison of outcomes between prior reports of PAHS and the patients in the present study is not possible, the MST of 391 days reported in our study is promising. When the outcomes for dogs with PAHS and HS of other anatomic locations were compared, dogs with PAHS lived significantly longer than did dogs with nonPAHS in our study. Additionally, the time to diagnosis was significantly longer in dogs with PAHS, compared with dogs with non-PAHS. Together, these data suggest that PAHS may follow a more indolent clinical course than HS of other anatomic locations. Alternatively, the longer survival time noted in the present study for dogs with PAHS may be a function of adequate local control, as $57.9 \%(11 / 19)$ of these dogs received definitive local treatment versus $25.8 \%$ (8/31) of dogs in the non-PAHS group. Furthermore, although the overall metastatic rate at diagnosis for both groups was approximately $70 \%$, metastasis involving visceral organs in dogs with PAHS was only $32 \%$ (6/19), compared with $71 \%(22 / 31)$ in dogs with non-PAHS. Thus, the improved outcome for the dogs in the PAHS group could be a function of the disease stage.

Previous reports ${ }^{4,7}$ evaluating HS in dogs identify anemia, thrombocytopenia, splenic involvement, hypoalbu- minemia, and the presence of metastatic disease at diagnosis as predictors of patient outcome. In univariate analysis, 2 factors were associated with a poorer prognosis when evaluating dogs with PAHS in the present study. The presence of metastatic disease at diagnosis was associated with a significantly shortened survival time, whereas concurrent administration of prednisone was associated with significantly shortened TTP for dogs with PAHS. Prior reports ${ }^{4,7}$ have indicated that once metastatic disease is evident in patients with HS, the clinical course is rapidly progressive. In the present study, the MST in dogs with PAHS with suspected metastatic disease at initial examination was 253 days, compared with 980 days in dogs with PAHS without evidence of metastatic disease. This suggests that the longterm prognosis for dogs with PAHS may be good when disease is detected prior to evidence of gross metastatic disease and definitive treatment is pursued.

The administration of prednisone has been evaluated for prognostic significance in dogs with HS receiving lomustine in the presence of gross evidence of disease, with no significant impact on survival time noted. ${ }^{7}$ In the present study, dogs with non-PAHS receiving concurrent administration of prednisone had a similar outcome, when compared with dogs that did not receive prednisone. It is possible that the impact of prednisone on the outcome of dogs with non-PAHS could not be assessed because of the relatively short survival time in these dogs. Alternatively, the administration of prednisone in dogs with PAHS may affect outcome in a disease-specific manner.

In multivariate analysis, dogs with PAHS in the present study that received concurrent prednisone treatment had an increased risk of death and tumor progression. This finding may reflect selection bias for dogs with more overt clinical signs and advanced stage at diagnosis, although the presence of metastatic disease was one of the factors controlled for in the multivariate analysis. It is important to note that the number of dogs receiving prednisone was small (5 [26\%] dogs), and the results suggesting that administration of prednisone has negative prognostic value may simply be a spurious finding. A prospective, randomized trial evaluating prednisone administration in dogs with PAHS would clarify its impact on outcome, and in vitro studies are necessary to determine whether prednisone has a direct effect on survival, proliferation, or chemoresistance in canine HS cells.

Interpretation of the results of the present study is limited by the small number of patients, the lack of standardized diagnostic and staging evaluation and treatments, and its retrospective nature. The diagnosis of HS is challenging despite advances in veterinary pathological techniques. Histopathology and immunohistochemical evaluation with antibodies directed against the CD18 marker are necessary for definitive diagnosis. In a recent study conducted by Wiley et al, ${ }^{c}$ cytologic evaluation alone for the diagnosis of HS was compared with histopathologic evaluation and immunohistochemical staining with CD18. Cytological diagnosis of HS was a specific (97\% [33/34]) but not sensitive test (44\% [4/9]), with an overall accuracy of $86 \%(37 / 43)$ and an $80 \%(4 / 5)$ positive predictive value. The authors concluded that cytologic evaluation alone is useful in patients in which there is a high suspicion for HS to confirm the diagnosis. ${ }^{c}$ 
Some of the patients in the present study were evaluated by use of cytologic evaluation alone and may have been incorrectly classified.

In the present report, efforts were made to exclude dogs with the hemophagocytic form of HS; however, definitive diagnosis of hemophagocytic HS requires immunohistochemical evaluation with antibodies against the CDlld marker, which was not assessed in this study. It is possible that some of the cases of non-PAHS were in fact hemophagocytic HS, which is believed to carry a poorer prognosis. ${ }^{1}$ Additionally, although all dogs in this study received treatment beyond pain management, the treatments administered were not standardized. Chemotherapy agents varied, although the majority of dogs received lomustine, which is the only drug that has reported activity against HS. ${ }^{6,7}$

Dogs with the periarticular form of HS may have a more favorable outcome than dogs with HS of other anatomic locations. This finding is independent of metastatic disease status, although the presence of metastatic disease in dogs with PAHS appears to be a negative prognostic indicator. Further prospective studies of dogs with PAHS are necessary to confirm these findings. Concurrent administration of prednisone in dogs with PAHS is associated with shorter TTP and increased risk of tumor progression and death. Additional studies are required to clarify the role prednisone may have, if any, in the progression of the disease.

a. MedCalc, version 10.2.0.0, MedCalc Software, Mariakerke, Belgium.

b. Varian Clinac 2100C, Varian Medical Systems Inc, Palo Alto, Calif.

c. Wiley J, Walton R, Kennedy D, et al. Comparison of cytology, flow cytometry using CD18, and histopathology with immunohistochemistry (CD18) for the diagnosis of canine histiocytic sarcoma (abstr), in Proceedings. 29th Annu Conf Vet Cancer Soc 2009;28.

\section{References}

1. Moore PF, Affolter VK, Vernau W. Canine hemophagocytic histiocytic sarcoma: a proliferative disorder of CDlld+ macrophages. Vet Pathol 2006;43:632-645.

2. Affolter VK, Moore PF. Localized and disseminated histiocytic sarcoma of dendritic cell origin in dogs. Vet Pathol 2002;39:74-83.

3. Craig LE, Julian ME, Ferracone JD. The diagnosis and prognosis of synovial tumors in dogs: 35 cases. Vet Pathol 2002;39:66-73.

4. Fidel J, Schiller I, Hauser B, et al. Histiocytic sarcomas in FlatCoated Retrievers: a summary of 37 cases (November 1998March 2005). Vet Comp Oncol 2006;4:63-74.

5. Schultz RM, Puchalski SM, Kent M, et al. Skeletal lesions of histiocytic sarcoma in nineteen dogs. Vet Radiol Ultrasound 2007;48:539-543.

6. Skorupski KA, Rodriguez CO, Krick EL, et al. Long-term survival in dogs with localized histiocytic sarcoma treated with CCNU as an adjuvant to local therapy. Vet Comp Oncol 2009;7:139-144.

7. Skorupski KA, Clifford CA, Paoloni MC, et al. CCNU for the treatment of dogs with histiocytic sarcoma. J Vet Intern Med 2007;21:121-126. 